\title{
HYPERSPECTRAL IMAGING: POTENTIAL IN NON-DESTRUCTIVE ANALYSIS OF PALIMPSESTS
}

\author{
Konstantinos Rapantzikos \\ Forth-Photonics \\ e-mail: rapantzikos@forth-photonics.gr
}

\author{
Costas Balas \\ Technical University of Crete $^{1} \&$ \\ Forth-Photonics ${ }^{2}$ \\ e-mail ${ }^{1}$ : balas@electronics.tuc.gr \\ e-mail ${ }^{2}$ balas@,forth-photonics.gr
}

\begin{abstract}
Palimpsests -twice written manuscripts- are of great interest since they may contain important hidden text underneath the visible one. Hyperspectral imaging may aid the expert to read the old script by enhancing the contrast between the under- and -overwriting. We present a hyperspectral imager (MUSIS), capable of acquiring 34 calibrated spectral bands in the range of $360-1150 \mathrm{~nm}$ (extended to $1550 \mathrm{~nm}$ when coupled with a photocathode tube). The potential of spectral imaging to improve the readability of manuscripts by employing common spectral analysis techniques is explored. Results on different manuscripts obtained by Principal Component Analysis (PCA) and Linear Spectral Mixture Analysis (LSMA) prove that hyperspectral imaging tools has the potential to become an indispensable tool for the analysis of old manuscripts.
\end{abstract}

\section{INTRODUCTION}

Hyperspectral imaging techniques have proven to be a powerful tool in the scientific analysis and documentation of paintings in the field of Art Research \& Conservation. Recently, spectral imaging was used for the inspection of palimpsests [1]. Palimpsests are twice written manuscripts dated from the medieval age. It was a common practice, particularly in ecclesiastical circles, to rub out an earlier piece of writing by means of washing or scraping it, in order to prepare it for a new text. The motive for making palimpsests seems to be largely economic, since reusing parchment was cheaper than preparing new skin. Another motive may have been directed by the desire of Church officials to "convert" pagan Greek script by overlaying it with the word of God. Modern historians are usually more interested in the older writings.

Easton et al. [1] were the first to capture and enhance the erased writings of the well known Archimedes palimpsest by multispectral methods. The system they propose is modeled on the VASARI illumination system that was developed at the National Gallery of London [2] for capturing digital images of artwork. Although, their findings are encouraging, they comment on the objectivity of the adopted method and the need for efficient and automatic ways to combine spectral bands in order to achieve high contrast between the upper (new) script and the script underneath (old).

In this paper we describe an integrated system for acquiring multispectral images and propose different techniques to present the rich information available from the spectral sensor in a readily interpretable manner. These techniques exploit the whole spectral cube (a stack of neighboring spectral bands) and reveal the spectral signatures of the contained materials. The core of the system is the MUSIS camera developed at ForthPhotonics. MUSIS enables the acquisition and display of multiple narrow-band images and allows for a fully resolved diffuse reflectance and/or fluorescence spectrum at each individual pixel. Generally, there are narrow spectral bands at which the maximum difference in the reflectance characteristics of each ink and parchment exists. Imaging at these bands will enable the maximization of the contrast between the two scripts. The collected data may be used for pigment analysis (spectrometry) or for spectral enhancement/visualization. In this work we focus on the ability of the system to identify different spectral signatures and combine them in order to enhance the erased script.

The rest of the paper is organized as follows: details on MUSIS are given in section 2. In section 3 we elaborate on two common spectral analysis methods. Experimental results and conclusions are given in sections 3 and 4 , respectively.

\section{HYPERSPECTRAL DATA ACQUISITION}

The MUSIS system developed by Balas [9, 8] at ForthPhotonics provides an optimum set-up for the acquisition of multiple spectral images by the coupling of imaging detectors with imaging monochromators. It is a hyperspectral imaging system, which is specialized for the in-situ, non-destructive analysis of Art \& Historic (A\&H) objects. The MUSIS imaging technology enables the integration of a plurality of imaging modes (reflectance, fluorescence) and spectral bands, ranging from UltraViolet (UV) to Near-Infrared (NIR) in one camera. The system enables the acquisition and display of multiple 
narrow-band images and a fully resolved diffuse reflectance and/or fluorescence spectrum at each individual pixel.

MUSIS displays in real time and captures images with an analysis of $1280 \times 960$ pixels. It can perform spectral imaging in 34 spectral bands from UV to NIR through a tunable optical filter, integrated to the detector, which enables the digital acquisition of the data. The tuning of the imaging wavelength is achieved by the displacement of the optical elements, which is performed with the aid of electromechanical manipulators, and is controlled from a PC via a microcontroller. The camera is sensitive in the spectral range 360nm (UV) $-1150 \mathrm{~nm}$ (extended to $1550 \mathrm{~nm}$-NIR- when coupled with a photocathode tube), which is achieved with an appropriate combination of a CCD optical detector and an infrared-tovisible imaging converter.

Specially developed software is employed for the control of the camera and monochromator as well as for the spectral image analysis. The system operates in two modes: the spectroscopy mode and the spectrometry mode. The former enables the random selection and real time visualization of desired spectral images, while the latter mode performs synchronized spectral scanning and image capturing and, finally, calculation of one full spectrum per image pixel. In both cases, a special calibration procedure [10] is executed before the imaging procedures, in order to compensate for the wavelength dependence of the response of the electro-optical parts of the system.

\section{HYPERSPECTRAL DATA ANALYSIS}

\subsection{Principal Component Analysis}

Selecting the most representative spectral bands in terms of information related to the different included scripts is crucial. Multispectral images bring an important quantity of information, which is redundant or complementary. If the value of one "feature" can be predicted precisely from the value of the others, then it is clearly redundant and can be dropped. PCA offers more than dropping features: PCA finds orthogonal linear combinations of a set of features (spectral bands) that maximize the variation contained within them, thereby displaying most of the original variation in a smaller number of dimensions. Hence, it transforms a multidimensional space to one of an equivalent (or less) number of dimensions by creating a new series of images (components) in which the axes of the new coordinate systems point in the direction of decreasing variance. In the transformed space the first dimension contains the most variability in the data, the second the second most, and so on.

This method is a classical technique from statistical pattern recognition and the related mathematics can be found in any related text book [3]. Given a threshold which characterizes the tolerated level of redundancy within the different bands, we obtain a number of complementary bands. Pixels with different spectral signatures appear more contrasted in the transformed than in the original bands.

\subsection{Linear Spectral Mixture Analysis}

LSMA has been widely investigated as a physically based image analysis process for handling the spectral mixture analysis of hyperspectral images $[6,7]$. It has been used to determine the relative abundances of materials that are depicted in hyperspectral imagery based on the material's spectral characteristics. Obviously, LSMA may aid the separation of spectrally different inks and parchment in the palimpsest case.

Generally, the presumed linear mixing model assumes no interaction between materials and a pixel is treated as a linear combination of signatures resident in the pixel with relative concentrations. The basic idea is the assumption of insignificant amount of multiple scattering between the different class types, i.e. each photon that reaches the sensor has interacted with just one class type. So received energy can be thought as a sum of the energies received from each class, which depends on the characteristics of the class type and the area covered by that type. In our analysis, the different classes correspond to different types of inks and the parchment. Hence, the measured spectral reflectance $r_{i}$ at each pixel is:

$$
r_{i}=\sum_{j} S_{i j} a_{j}
$$

where $S_{i j}$ is the matrix of selected spectral signatures and $a_{j}$ is the percentage abundance of the material in class $j$ at a given pixel. As indicated in [1], a simple modification is required to adapt the model to the case of ink and parchment because their interaction is actually multiplicative. Evaluating the logarithm of the pixel values and the spectral signatures converts the case to a linear one.

A main disadvantage of the LSMA is the need for $a$ priori knowledge of endmember signatures resident in the hyperspectral images, which are often difficult to be acquired them in practice. This is a major disadvantage when dealing with palimpsests, since the underwriting is not always clearly visible in any band and it is difficult to select the corresponding endmembers. Another disadvantage is that it is not always possible to select pixels that are representative of single classes for the entire leaf of the manuscript, since the spectrum of the parchment or a given ink may vary across the page [1].

\section{RESULTS}

Several leafs of palimpsests have been acquired by the MUSIS system during the last years. Due to lack of space 
we present results on two different, but representative cases. The first one (Patmos) is hold in the monastery of St. John Theologos in Patmos. The overwriting is a part of a manuscript entitled "Tetraevaggelio" aged from $11^{\text {th }}$ century $\mathrm{AD}$, while the underwriting is part of an unknown text aged from 1282 AD. As indicated before, the overwriting consists of two different types of ink. The second palimpsest (Agio Oros) is hold in the monastery of Vatopedi in Agio Oros. The older script, entitled "Porphyrogennetus", is dated from the $10^{\text {th }}$ to $11^{\text {th }}$ century $\mathrm{AD}$, while the newer one, entitled "Ymnoi stin Theotokon" ("Madonna Anthems"), is dated from the $13^{\text {th }}$ century AD.

In order to get a grip of the possible measures and the enhancement ability that can be obtained from a hyperspectral cube we show six different bands of a detail from the Patmos palimpsest acquired at different wavelengths (Fig. 1b). Notice how the older script becomes transparent as the wavelength increases, while the overwriting is retained longer. The spectral curve of a single pixel that belongs to the parchment, the upper (black) and the underwriting (gray) respectively is shown in Fig. 1a.

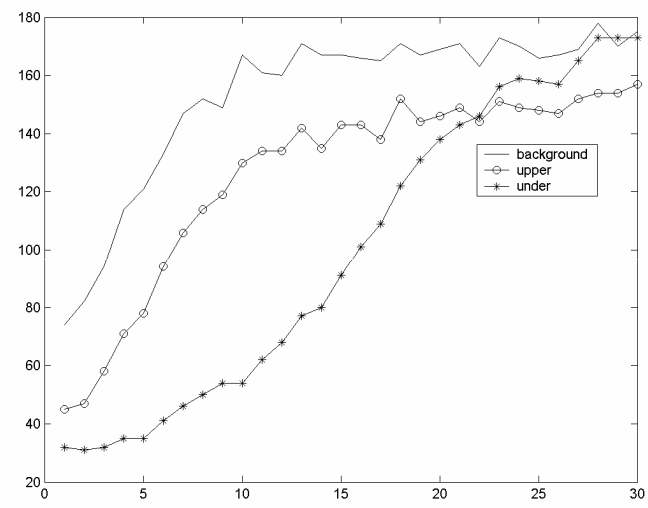

(a)

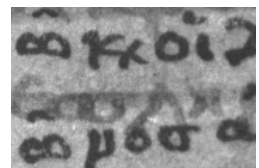

$420 \mathrm{~nm}$

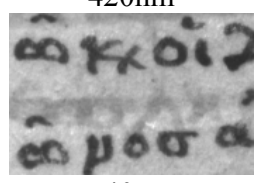

$540 \mathrm{~nm}$

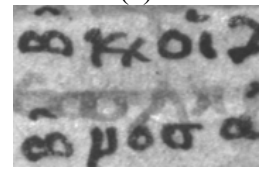

$460 \mathrm{~nm}$

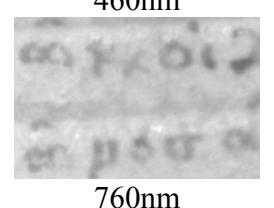

(b)

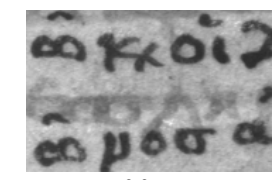

$500 \mathrm{~nm}$

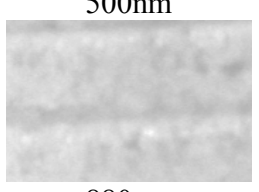

$880 \mathrm{~nm}$
Fig. 1 (a) spectral profile of a pixel belonging to the background, overwriting and underwriting respectively; (b) 6 different spectral bands of a palimpsest region

\subsection{PCA Pseudocoloring}

A common method to present the principal component images in a pseudocolor display is to map the first three PCs (or another set of PCs) into an RGB image. Another strategy is to select two of the PCs and use the first into two of the three RGB channels and the second one into the remaining one [11]. Such a selection depends on the information that we want to enhance when combining the transformed bands.

To compare the discriminating power of a spectral images combination against an RGB image, we reduce the number of bands to three. For this, we transform the whole range of spectral bands using PCA and obtain the first three bands as explained above. Fig. 2 illustrates a selected region of the Patmos palimpsest. This manuscript contains two scripts, an old and a new one, with the new one being comprised from two types of ink. Fig. 1a shows the region in RGB. Notice the different colors that correspond to the old/new scripts and the different kinds of ink. The reddish letters (light and dark) belong to the new, while the light brown to the older one. The parchment has a similar brownish color and makes the distinction difficult. The fluorescence UV band reveals both scripts as shown in Fig. 1b, but the differentiation of the different inks in terms of intensity is still difficult. The three PCA bands (Fig. 2cde) enhance or suppress regions of the manuscript with different spectral signatures and give the expert the opportunity to examine efficiently the content of the palimpsest. The pseudocolored image (Fig. 1f) is generated by replacing the red band with the second $\mathrm{PC}$ and the green and blue with the third one $\left(P_{2} P_{3} P_{3} \rightarrow R G B\right)$.

\subsection{Spectral Unmixing}

LSMA requires the selection of endmembers for each spectral class. Selecting them for the Patmos case is easy, while the opposite holds for the Agio Oros. The palimpsest's degradation is heavier and the underwriting is barely visible. Endmembers for the underwiting were chosen after proper inspection of this manuscript with the aid of an expert.

Figure 3 illustrates the potential of PCA and LSMA to enhance the readability of the palimpsests using a large range of spectral information. The linear spectral unmixing results are shown in Fig. $3 \mathrm{~cd}$. We run PCA on the available hyperspectral info and keep the first three PCs. After appropriate mapping to RGB channels (see section 4.1), we generate the pseudocolored images in Fig. 3ef. Fig. 3ab show the original RGB images for the selected regions. For both the PCA and LSMA to RGB mapping we used the following reasoning: the band that highlights the underwriting is assigned to the red channel, while both green and blue channels are replaced by the same band that highlights more the background. 


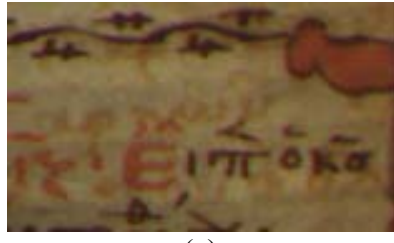

(a)

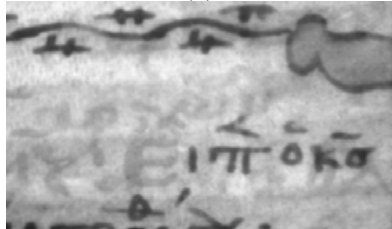

(c)

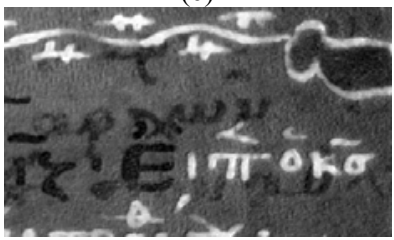

(e)

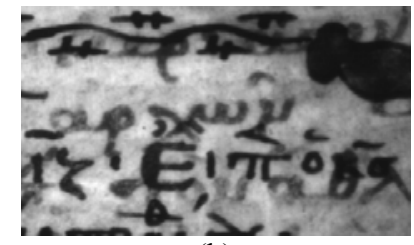

(b)

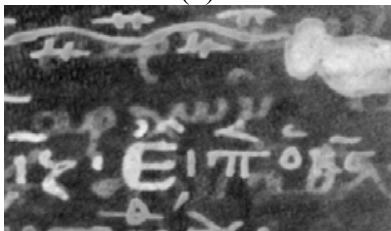

(d)

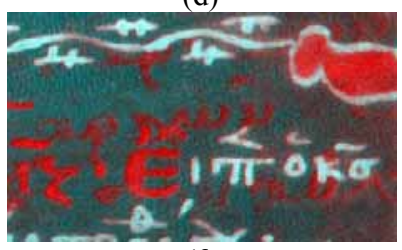

(f)
Fig. 2 (a) RGB; (b) fluorescence UV;(c)-(e) first three PCA bands; (f). Pseudocolored image from PCA bands

Remarkably enough, the RGB images contain almost no information (in terms of color) about the underwritings. PCA and LSMA perform equally well for the Patmos case, as seen from the red color assigned to the older scripts (Fig. 3ce). The result of LSMA in Fig. 3d is more or less confusing about the presence of the older script, while PCA (Fig. 3f) performs better in differentiating the older script from the newer one. Obviously, the selected endmembers do not represent well the different types of materials present in the manuscript.

\section{CONCLUSIONS}

An integrated system for hyperspectral acquisition and processing of palimpsests is presented. An elaboration on the potential of two common spectral analysis techniques, namely PCA and LSMA, to separate the spectral signatures of parchment, overwriting and underwriting is attempted. The results are quite promising.

Further experimentation is needed in order to reach a more mature decision about the specific algorithmic steps required for the analysis of old manuscripts, since the variability of destruction (age, burn etc.) makes their intra- spectral differentiation difficult. We also plan to design an automatic method for combining the different spectral bands in order to enhance a target signature. Use of PCA as a method to automatically select endmembers for the LSMA is also a topic of our research.

\section{REFERENCES}

[1] Easton R.L., Knox K.T., Christens-Barry W.A., Multispectral imaging of the Archimedes Palimpsest, Proc AIPR'03, IEEE, 2003

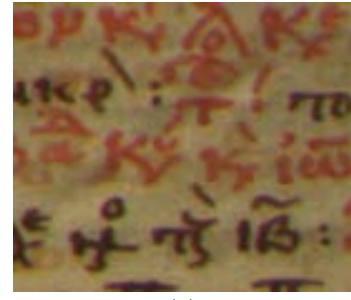

(a)

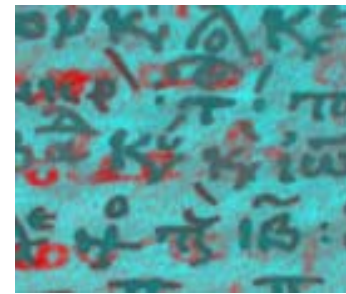

(c)

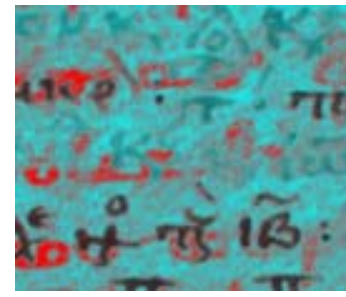

(e)

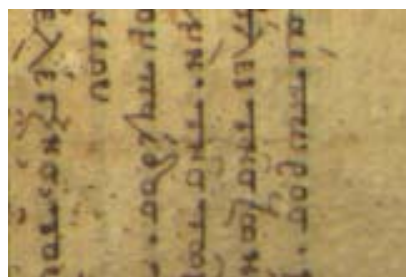

(b)

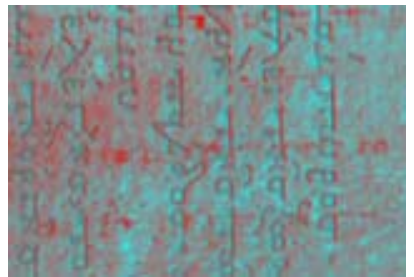

(d)

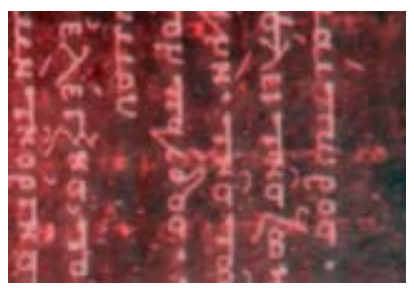

(f)
Fig. 3 (a)-(b) RGB images; (c)-(d) LSMA resuls; (e)-(f) PCA results

[2] Martinez K., Cupitt J., Saunders D., Pillay R., Ten years of art imaging research, Proc. IEEE 90 (1), pp. 28-41, 2002

[3] Duda R.O., Hart P.E., Stork D.G., Pattern Classification, John Wiley and Sons, New York, 2001.

[4] Campbell, B.J., Introduction to Remote Sensing, second edition, The Guilford Press, New York, NY, 1996

[5] Bajcsy P., Groves P., Methodology for hyperspectral band selection, Photogrammetric Engineering and Remote Sensing journal, vol. 70, no. 7, pp. 793-802, Jul 2004.

[6] Heinz D., Chang C.-I., Fully constrained least squares linear spectral mixture analysis method for material quantification in hyperspectral imagery, IEEE Trans. Geosci. Remote Sensing, vol. 39, no. 3, Mar. 2001.

[7]. Keshava N., Mustard J.F., Spectral Unmixing, IEEE Signal Processing Magazine, vol. 19, no. 1, Jan 2002.

[8]. Anglos D., Balas C., Fotakis C., Laser spectroscopic and optical imaging techniques in chemical and structural diagnosis of painted artworks, Am. Lab., pp. 60-67, Oct 1999.

[9]. Balas C., An imaging method and apparatus for the nondestructive analysis of paintings and monuments, International Patent App., PCT/GR00/00039.

[10]. Balas C., A novel optical imaging method for the early detection, quantitative grading and mapping of cancerous and precancerous lesions of cervix, IEEE Trans. Biomed. Eng, vol. 48, pp. 96-104, 2001

[11]. Typ S.J., Konsolakis A., Diersen D.I., Olsen R.C., Principal-components-based display strategy for spectral imagery, IEEE Trans. On Geosci. And Remote Sens., vol. 41, no. 3, Mar 2003. 